\title{
Single-cell imaging and transcriptomic analyses of endogenous cardiomyocyte dedifferentiation and cycling
}

\author{
Yiqiang Zhang ${ }^{1,2,3}$, Nuria Gago-Lopez 1,2,3, Ning Li ${ }^{1,2,3,4}$, Zhenhe Zhang ${ }^{1,2,3}$, Naima Alver ${ }^{1,2,3}$, Yonggang Liu ${ }^{1,2,3}$,
} Amy M. Martinson ${ }^{2,3,5}$, Avin Mehri ${ }^{1,2,3}$ and William Robb MacLellan ${ }^{1,2,3,6}$

\begin{abstract}
While it is recognized that there are low levels of new cardiomyocyte (CM) formation throughout life, the source of these new CM generates much debate. One hypothesis is that these new CMs arise from the proliferation of existing CMs potentially after dedifferentiation although direct evidence for this is lacking. Here we explore the mechanisms responsible for CM renewal in vivo using multi-reporter transgenic mouse models featuring efficient adult CM (ACM) genetic cell fate mapping and real-time cardiomyocyte lineage and dedifferentiation reporting. Our results demonstrate that non-myocytes (e.g., cardiac progenitor cells) contribute negligibly to new ACM formation at baseline or after cardiac injury. In contrast, we found a significant increase in dedifferentiated, cycling CMs in post-infarct hearts. ACM cell cycling was enhanced within the dedifferentiated CM population. Single-nucleus transcriptomic analysis demonstrated that CMs identified with dedifferentiation reporters had significant down-regulation in gene networks for cardiac hypertrophy, contractile, and electrical function, with shifts in metabolic pathways, but up-regulation in signaling pathways and gene sets for active cell cycle, proliferation, and cell survival. The results demonstrate that dedifferentiation may be an important prerequisite for CM proliferation and explain the limited but measurable cardiac myogenesis seen after myocardial infarction (MI).
\end{abstract}

\section{Introduction}

The potential of cardiomyocytes (CMs) to proliferate is tightly developmentally controlled. The mammalian heart grows by hyperplasia during fetal life but this proliferative potential is lost in the adult. Neonatal CMs retain some proliferation capacity and can even regenerate lost myocardium after injury, but this ability is lost by 7 days after birth $^{1-4}$. Adult CM (ACM) growth is typically hypertrophic; however, there is a very low, but measurable rate of new CM formation in adult hearts ${ }^{1,5-8}$. Although it has been much debated on the source of this proliferation and

\footnotetext{
Correspondence: Yiqiang Zhang (yzhang@cardiology.washington.edu) (kenzhangyq@hotmail.com) or William Robb MacLellan

(WRMacLellan@cardiology.washington.edu)

'Division of Cardiology, Department of Medicine, University of Washington, Seattle, WA, USA

${ }^{2}$ Center for Cardiovascular Biology, University of Washington, Seattle, WA, USA Full list of author information is available at the end of the article.
}

whether it is restricted to a subset of ACMs, the magnitude, an annual renewal rate of $\sim 0.5-1 \%$ has now been accepted by most investigators ${ }^{6,8-11}$. Given the low rate of overall ACM renewal, detecting CM cell cycle progression especially cell division is challenging but crucial for future studies targeting endogenous $\mathrm{CM}$ regeneration. Recent strategies to quantitate new ACMs based on heavy isotope labeling appear to be more accurate than standard histology, but they require specialized expertise and equipment and are limited both in their throughput and ability to be combined with mechanistic studies. We sought to develop a simplified, high-throughput system that provides enhanced accuracy and facilitates the study of the cellular sources and mechanisms underlying CM renewal in adult mice.

Theoretically, new CMs could arise from the differentiation of resident cardiac progenitor cells (CPCs) or by

\section{(c) The Author(s) 2019}

(c) (i) Open Access This article is licensed under a Creative Commons Attribution 4.0 International License, which permits use, sharing, adaptation, distribution and reproduction c. in any medium or format, as long as you give appropriate credit to the original author(s) and the source, provide a link to the Creative Commons license, and indicate if changes were made. The images or other third party material in this article are included in the article's Creative Commons license, unless indicated otherwise in a credit line to the material. If material is not included in the article's Creative Commons license and your intended use is not permitted by statutory regulation or exceeds the permitted use, you will need to obtain permission directly from the copyright holder. To view a copy of this license, visit http://creativecommons.org/licenses/by/4.0/. 
the proliferation of pre-existing $\mathrm{CMs}^{12,13}$. CPCs are essential for normal cardiac development, but their role, if any, in adult hearts is disputed and uncertain ${ }^{4,14-24}$. Recent genetic cell fate tracking and clonal analyses demonstrated that new myocytes likely arise from preexisting CMs. However, incomplete genetic labeling of ACMs in previous Cre/LoxP models and inefficient gene recombination inherent in "mosaic analysis with double markers" model have made the findings inconclusive ${ }^{5,8}$. In order to fully characterize endogenous myocyte renewal, we developed a new bi-transgenic $\alpha \mathrm{MHC}-\mathrm{MCM} ; \mathrm{RFP}^{\mathrm{fl}} /$ GFP system that has improved CM fate mapping ${ }^{25,26}$.

Dedifferentiation is a regressive process where specialized cells or tissues regain primitive phenotypes-this is critical for repair and regeneration in many lower vertebrates. While ACMs are known to be able to dedifferentiate and re-differentiate both in cell culture and when transplanted into post-infarct myocardium ${ }^{27-31}$, whether this happens in vivo and how to accurately quantify the magnitude of myocyte dedifferentiation is unknown. To address these limitations, we created a cardiac nucleusspecific reporter transgenic mouse $T g(M y h 6-$ $H 2 B B F P 6 x H i s)$ referred to as the blue fluorescent protein (BFP) model. This BFP mouse model enabled the highthroughput quantification of ACMs and their dedifferentiation. BFP signal was highly expressed in ACMs but reduced in dedifferentiated ACMs and immature myocytes, such as those from neonatal hearts. When our BFP mice are bred to bi-transgenic $\alpha \mathrm{MHC}-\mathrm{MCM} ; \mathrm{RFP}^{\mathrm{fl}} / \mathrm{GFP}$ mice, the new triple transgenic $\alpha M H C-M C M ; R^{2} P^{f l} / G F P$; BFP mice provide a genetic model to visualize and quantitate dedifferentiated CMs in vivo ${ }^{5,8,12,18,19,21,29-33}$. Using these novel transgenic models, we demonstrated that $\mathrm{CM}$ dedifferentiation occurs after cardiac injuries, and is associated with the enhanced ACM cycling in postinfarct hearts. Massive parallel single-nucleus RNA-seq (snRNA-seq) analysis revealed novel transcriptomes in the subset of CMs expressing specific transgene reporters consistent with dedifferentiation and active cell cycling. This model provides a useful tool to study the mechanisms controlling endogenous myocardial regeneration in injured hearts by combining high-throughput single-cell imaging and transcriptomic analyses.

\section{Results}

\section{Minimal contribution of non-myocyte pools to} cardiomyocyte renewal in post-infarct hearts

To determine the potential contribution of nonmyocyte populations, including putative resident CPCs to $\mathrm{CM}$ renewal ${ }^{1,5,8,18,19,21,29,31}$, we generated a bitransgenic $\alpha \mathrm{MHC}-\mathrm{MCM} ; \mathrm{RFP}^{\mathrm{fl}} / \mathrm{GFP}$ mouse model by cross-breeding $\alpha \mathrm{MHC}-\mathrm{MCM}$ mouse with Rosa26-mT/ $\mathrm{mG}$ reporter mouse (the latter referred to as $\mathrm{RFP}^{\mathrm{fl}} / \mathrm{GFP}$ mouse for its dual-color reporters in red and green fluorescences) (Fig. 1a; and Supplementary Fig. S1a) ${ }^{25,26}$. This bi-transgenic mouse model has a tamoxifen-inducible, CM-specific GFP signal that is superior to previously reported systems ${ }^{8,25,26,29}$. More than $98 \%$ of $\mathrm{CMs}$ in tamoxifen-treated bi-transgenic adult mice irreversibly switched from RFP to GFP expression (Fig. 1b, c). Immunostaining revealed the co-expression of GFP signal with CM markers, such as $\alpha$-myosin heavy chain $(\alpha M H C)$, Tropomyosin, $\alpha$-sarcomeric actinin $(\alpha-\mathrm{SA})$, and troponin I (cTnI) (Supplementary Fig. S1b, c). After tamoxifen treatment, over $98 \% \mathrm{ACMs}$ were $\mathrm{GFP}^{+}$; and there was a minor portion of CMs that co-expressed GFP and RFP $(<1 \%)$, likely due to asymmetric gene recombination ${ }^{34}$ in ACMs that were binucleated (Fig. 1c). There was a barely detectable $\mathrm{GFP}^{+}$population $(<0.03 \%)$ among small nonmyocytes, confirming that gene recombination was specific to ACMs, without leaky labeling of putative nonmyocytes, such as CPCs or committed cardiac precursors. Therefore, the GFP population specifically identified the vast majority of pre-existing ACMs in tamoxifen-treated bi-transgenic mice.

To determine if cardiac differentiation of any putative resident $\mathrm{CPCs}$ among the non-myocyte pool contributed to ACM renewal in post-infarct hearts, we induced myocardial infarction (MI) in tamoxifen-treated $\alpha \mathrm{MHC}$ $\mathrm{MCM} ; \mathrm{RFP}^{\mathrm{fl}} / \mathrm{GFP}$ adult mice and followed them for the appearance of "new" RFP-positive cardiac myocytes. There was no significant change in the percentage of $\mathrm{GFP}^{+}$cells among CMs up to 3 months after MI compared to sham-operated hearts (96.5\% in MI versus $97.9 \%$ in Sham) (Fig. 1d). The amount of $\mathrm{RFP}^{+} \mathrm{CMs}$ remained minimal, and showed no significant difference between sham and post-infarct hearts. To track ACM cell cycle activity, we labeled cycling cells in sham or post-MI bitransgenic mice for 3.5 weeks via drinking water containing 5-bromo-2'-deoxyuridine (BrdU). $\mathrm{BrdU}^{+} \mathrm{GFP}$ ACMs were significantly increased in post-infarct hearts compared to sham hearts as revealed by ImageStream, a multispectral, microscopy imaging-based flow cytometry analysis (Fig. 1e). No RFP ${ }^{+} \mathrm{BrdU}^{+} \mathrm{ACMs}^{-}$were detected in either post-MI or sham hearts (data not shown). These results are consistent with the notion that noncardiomyocyte pools do not contribute to ACM renewal in post-infarct hearts and suggest that if new myocytes are formed they arise from pre-existing $\mathrm{CMs}^{8}$.

\section{Generation of a cardiac-specific nuclear BFP reporter mouse to visualize myocyte maturity and dedifferentiation}

To track CMs with a real-time reporter of CM maturity, we generated an ACM nucleus-specific transgenic BFP mouse model. The BFP gene was fused in-frame to histone $H 2 B$ gene under the control of cardiac-specific aMHC (Myh6) promoter. Therefore, only ACMs expressed BFP (Fig. 2a, b; and Supplementary Figs. S2 and 


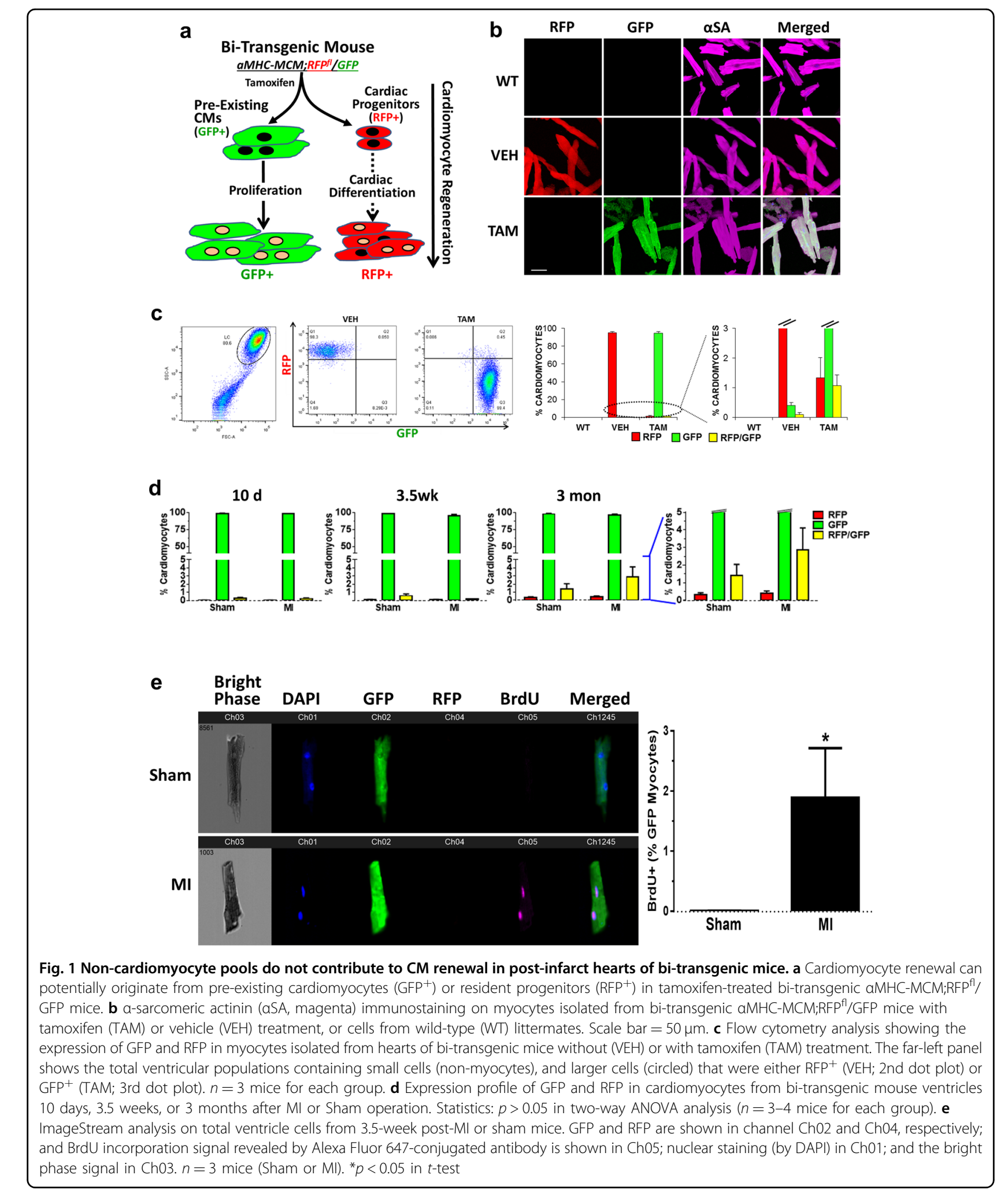

S3). $\mathrm{BFP}^{+}$nuclei also demonstrated strong co-expression of pericentriolar material 1 (PCM1) which has been used to identify ACM nuclei previously (Fig. 2c) ${ }^{11}$. Transgene expression did not adversely affect cardiac development or growth, and transgenic mice had normal cardiac histology and function (Supplementary Fig. S4). 

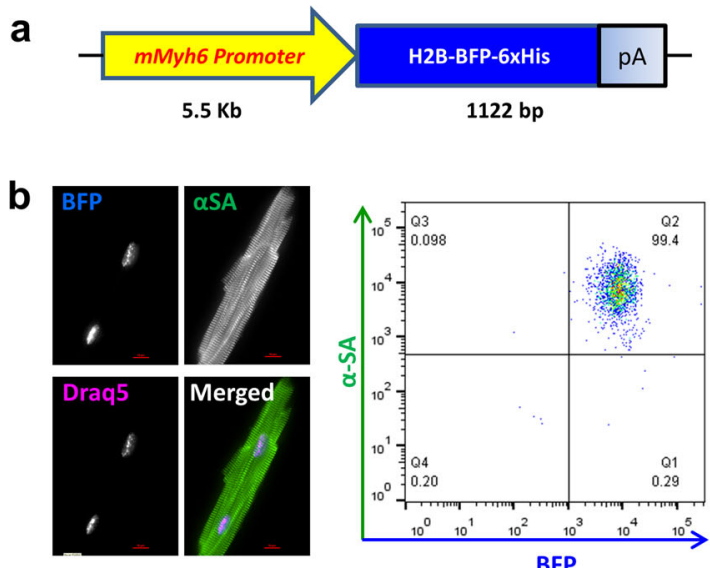

1122 bp

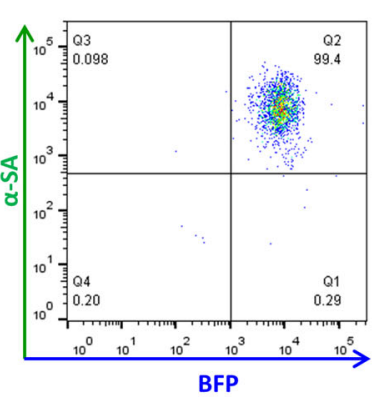

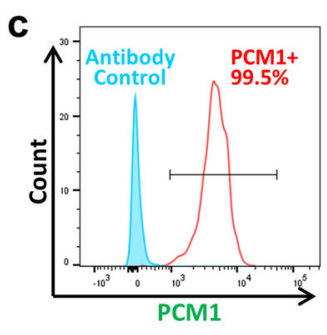

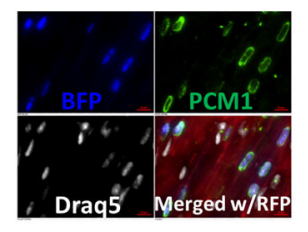

BFP Intensity in ACM

\section{dedifferentiation cell culture}

e
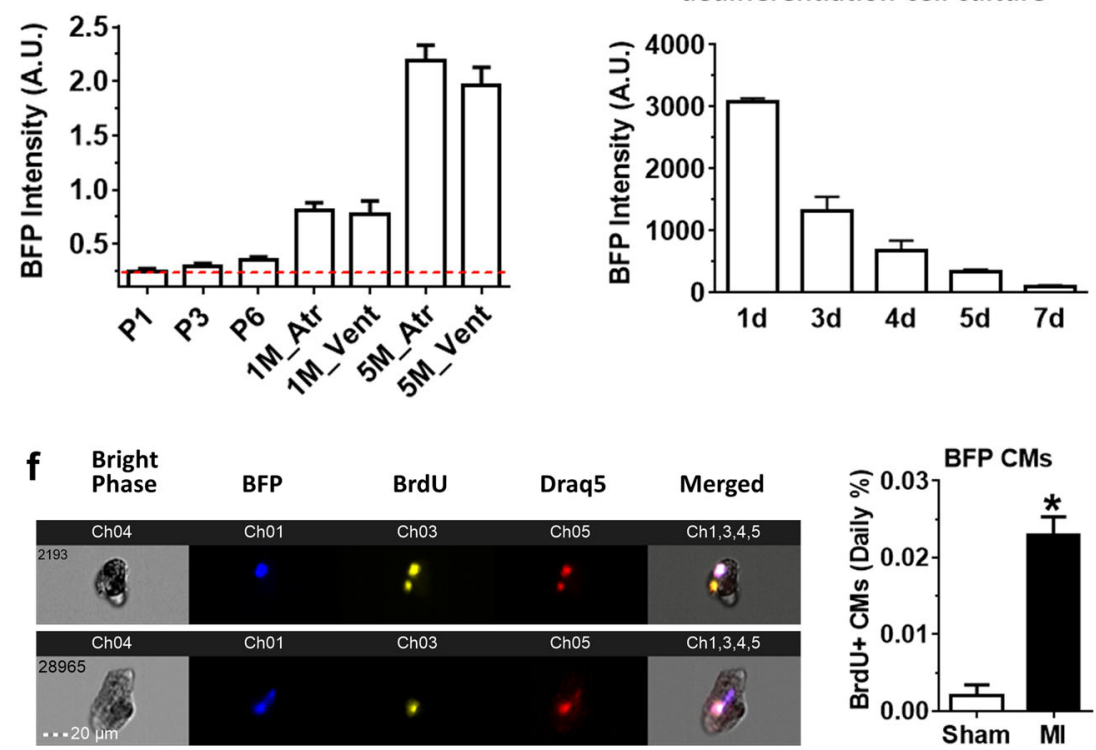

Fig. 2 Creation of cardiomyocyte nucleus-specific transgenic BFP mice. a Gene construct for transgenic cardiac nuclear blue fluorescent protein (BFP) reporter mouse. A full-length mouse a-MHC (mMyh6) promoter drives an in-frame fusion gene containing H2B, BFP, and a tag of six histidines (6xHis). Scale bar $=10 \mu \mathrm{m}$. b Confocal images (left panels) and flow cytometry dot plot (right panel) showing cardiomyocyte nucleus-specificity of BFP signal. Native blue fluorescent signal from BFP transgene was found in cardiomyocytes identified by a-sarcomeric actinin (a-SA, green); nuclei were stained by Draq5 (magenta). Scale bar $=10 \mu \mathrm{m}$. For flow cytometry analysis, samples were gated on Draq5 $5^{+}$population and a-SA (Alexa Fluor 488) and native BFP signal was analyzed. c Upper panel: Flow cytometry histogram showing the expression of pericentriolar material 1 (PCM1) (Alexa Fluor 488) in $\mathrm{BFP}^{+}$population in the total heart nuclei preparations. $98.8 \pm 0.9 \% \mathrm{BFP}^{+}$nuclei are $\mathrm{PCM}^{+}(n=3$ mice). Lower panel: Confocal image showing the expression of PCM1 (green) and native BFP signal (blue) in ventricular tissue of transgenic mouse carrying BFP and Rosa26-mT/mG (RFPflGFP) transgenes. RFP expressed ubiquitously in all cells. Nuclei were stained with Draq5 (pseudo-white). Scale bar $=100 \mu \mathrm{m}$. $\mathbf{d}$ Native BFP signal intensity normalized to DNA content determined by Draq5 staining in heart tissues. $p<0.0001$ with one-way ANOVA test. $n=3,4,3,3$, and 4 mice for P1, P3, P6, 1 month, and 5 months ages, respectively. e BFP signal determined by live cell imaging during adult CM dedifferentiation in cell culture. $p<0.0001$ with one-way ANOVA test. $N=300-500$ cells from three cell cultures. $\mathbf{f}$ ImageStream analysis of total ventricular cell preparations. Left panels: example BrdU ${ }^{+}$BFP cardiomyocytes. BFP signal is shown in Ch01, BrdU incorporation signal in Ch03, nuclear staining (by Draq5) in Ch05, and the bright phase signal in Ch04. ${ }^{*} p<0.01$ in t-test ( $n=3$ mice; Sham or MI)

Approximately $35 \%$ of total ventricular nuclei were $\mathrm{BFP}^{+}$ when assayed by either imaging native BFP fluorescent signal, or by immune reactivity to BFP and polyhistidinetag proteins (Supplementary Fig. S5). BFP signal was expressed in over $99 \%$ ACMs identified by the expression of $\alpha$-SA or cardiac Troponin T (cTnT) (Fig. 2b; and Supplementary Figs. S2, S3, and S5). Analyses of a panel of major organs further confirmed the expression of BFP was specific to CMs (Supplementary Figs. S6 and S7). Comparable $\mathrm{BFP}^{+}$populations were seen in the ventricles of 
young (1 month old) and adult (5 months old) hearts (Supplementary Fig. S8). Importantly, the BFP signal was developmentally regulated: minimally expressed in early neonatal CMs, but significantly augmented in ACMs (Fig. 2d; and Supplementary Fig. S9a and b). When isolated BFP ACMs were subjected to dedifferentiation cell culture conditions, BFP signal reduced rapidly within a week (Fig. 2e; and Supplementary Fig. S9c), consistent with the reduced $\alpha \mathrm{MHC}$ expression in dedifferentiated $\mathrm{CMs}^{29}$. Therefore, BFP signal could potentially be used as a surrogate to visualize CM maturity, and BFP signal reduction in ACMs may reflect their dedifferentiation.

To quantify ACM cycling in this model, we treated sham-operated or post-MI transgenic BFP mice with BrdU for 3.5 weeks. Approximately $30 \% \mathrm{BFP}^{+}$myocytes were lost in post-infarct hearts compared to sham as evaluated by ventricular $\mathrm{BFP}^{+}$nuclei (Supplementary Fig. S10). In surviving myocytes, there was a significant increase (9.2-fold) of $\mathrm{BFP}^{+} \mathrm{BrdU}^{+}$cells in post-MI hearts compared to sham mice (Fig. 2f). This labeling rate was comparable to previous isotope labeling studies when converted to similar pulsing period: $0.47 \%$ of all $\mathrm{CMs}$ in the whole-ventricle in our single-cell imaging analysis versus $1.2 \%$ specifically in the infarct border zone measured with multi-isotope imaging mass spectrometry (MIMS) analysis ${ }^{8}$.

\section{Increased dedifferentiated ACMs in post-infarct multi- reporter tri-transgenic mice}

Next, we sought to identify ACM dedifferentiation in vivo by creating a tri-reporter mouse model. By crossbreeding the mouse model for permanent GFP labeling in CMs ( $\alpha M H C-M C M ; R^{2} P^{f l} / G F P$ model) with the mice that express a CM-specific, maturity/dedifferentiation reporter (BFP model), we created a tripletransgenic mouse model. In this model, dedifferentiation of pre-existing $\mathrm{ACMs}$ would be identified as $\mathrm{GFP}^{+} \mathrm{BFP}^{\text {low }}$ (or $\mathrm{GFP}^{+} \mathrm{BFP}^{-}$) cells (Fig. 3a).

After tamoxifen treatment, over $99 \% \mathrm{GFP}^{+} \mathrm{CMs}$ were $\mathrm{BFP}^{+}$in tri-transgenic mice, and $\mathrm{RFP}^{+} \mathrm{BFP}^{+}$cells were minimal among ACMs (Fig. 3b). After MI, there was no significant change in GFP and RFP expression among $\mathrm{BFP}^{+} \mathrm{ACMs}$ when compared to those in sham-operated hearts, consistent with our previous results (Fig. 3c). In total ventricular cells, only $\sim 0.02 \%$ were $\mathrm{RFP}^{+} \mathrm{BFP}^{+}$at baseline, which might represent the minimal $\mathrm{RFP}^{+} \mathrm{ACMs}$ that had failed to undergo Cre/LoxP gene recombination. The $\mathrm{RFP}^{+} \mathrm{BFP}^{+}$population remained minimal and not different between post-MI or sham hearts (Fig. 3c). Although ACMs suggestive of dedifferentiation have been shown in previous studies, definitive in vivo proof has been lacking. As shown in Fig. 4a, there was approximately a three-fold increase in the rare $\mathrm{GFP}^{+} \mathrm{BFP}^{\text {low }}$ population in post-MI hearts compared to sham hearts.
The forward and side scatter indices (FSC and SSC, respectively) for $\mathrm{GFP}^{+} \mathrm{BFP}^{\text {low }}$ cells in post-MI hearts were significantly lower than those in sham-operated hearts (Fig. 4b, c), suggesting they were smaller and had rounder morphology.

Definitively identifying dedifferentiated $\mathrm{CM}$ is problematic, as no consensus exists on the specific molecular signatures. To identify genes that were unique to the dedifferentiated state, we performed whole-transcriptome analysis on in vitro dedifferentiated CM, embryonic CMs, normal ACMs or hypertrophic ACMs. Dedifferentiated CMs displayed a distinct transcriptional profile (Supplementary Fig. S11). Dedifferentiation-specific genes were defined as those upregulated in dedifferentiated CMs compared to normal ACMs, excluding genes that were more highly expressed in embryonic CMs or hypertrophic ACMs (Supplementary Table S1). We then used this molecular signature to compare $\mathrm{GFP}^{+} \mathrm{BFP}^{\text {high }}$ (normal $\mathrm{ACMs}$ ) to $\mathrm{GFP}^{+} \mathrm{BFP}^{\text {low }}$ cells in post-infarct hearts. Consistent with in vitro results, $\mathrm{GFP}^{+} \mathrm{BFP}^{\text {low }}$ cells expressed significantly higher levels of these dedifferentiation genes, including Fos-like antigen 1 (Fosl1), myelocytomatosis oncogene $(M y c), \quad$ S100 calcium-binding protein A6 (S100a6), SRY-box containing gene 4 (Sox4), and thymosin beta 10 (Tmsb10). In addition, cardiac marker $M y h 7$ was significantly higher than $M y h 6$ in $\mathrm{GFP}^{+} \mathrm{BFP}^{\text {low }}$ myocytes although both transcripts were down-regulated (Fig. 4d). Thus, $\mathrm{GFP}^{+} \mathrm{BFP}^{\text {low }}$ cells in tri-transgenic hearts are morphologically and molecularly similar to dedifferentiated $\mathrm{CM}^{27,29-31}$.

\section{Dedifferentiated CMs contributed to active cycling ACMs in post-infarct hearts}

Dedifferentiated ACMs in tissue culture demonstrate increased cell cycling and are capable of proliferation ${ }^{29,30}$. Given the increased cycling of GFP myocytes in post-MI bi-transgenic mice (Fig. 1e) and that $\mathrm{GFP}^{+} \mathrm{BFP}^{\text {low }}$ cells expressed higher cell cycle genes, such as $C c n b 1$ and $C d k 2$ (Fig. 4d), we hypothesized that BFP ${ }^{\text {low }}$ ACMs might have enhanced cell cycling and proliferation activity. Flow cytometry analysis revealed similar levels of $\mathrm{BrdU}^{+}$GFP CMs in post-MI hearts of tri-transgenic mice compared to post-MI bi-transgenic hearts, which were both significantly higher than that in sham-operated hearts (Fig. $5 \mathrm{a})$. However, the rate of cycling was $\sim 50 \%$ higher in the dedifferentiated $\mathrm{ACM}$ subpopulation $\left(\mathrm{GFP}^{+} \mathrm{BFP}^{\text {low }} ; 2.2 \%\right)$ compared to normal, mature $\mathrm{ACMs}\left(\mathrm{GFP}^{+} \mathrm{BFP}^{\text {high}}\right.$; 1.49\%) (Fig. 5b). To identify regional differences in CMs dedifferentiation and cell cycle activity in post-infarct hearts, we performed high-content imaging analysis of whole-ventricular sections (Fig. 5c, d). The BFP signal in cycling $\mathrm{GFP}^{+}$myocytes was barely detectable in periinfarct zones, with reduced expression in border zones, while expression remained at normal levels in remote 

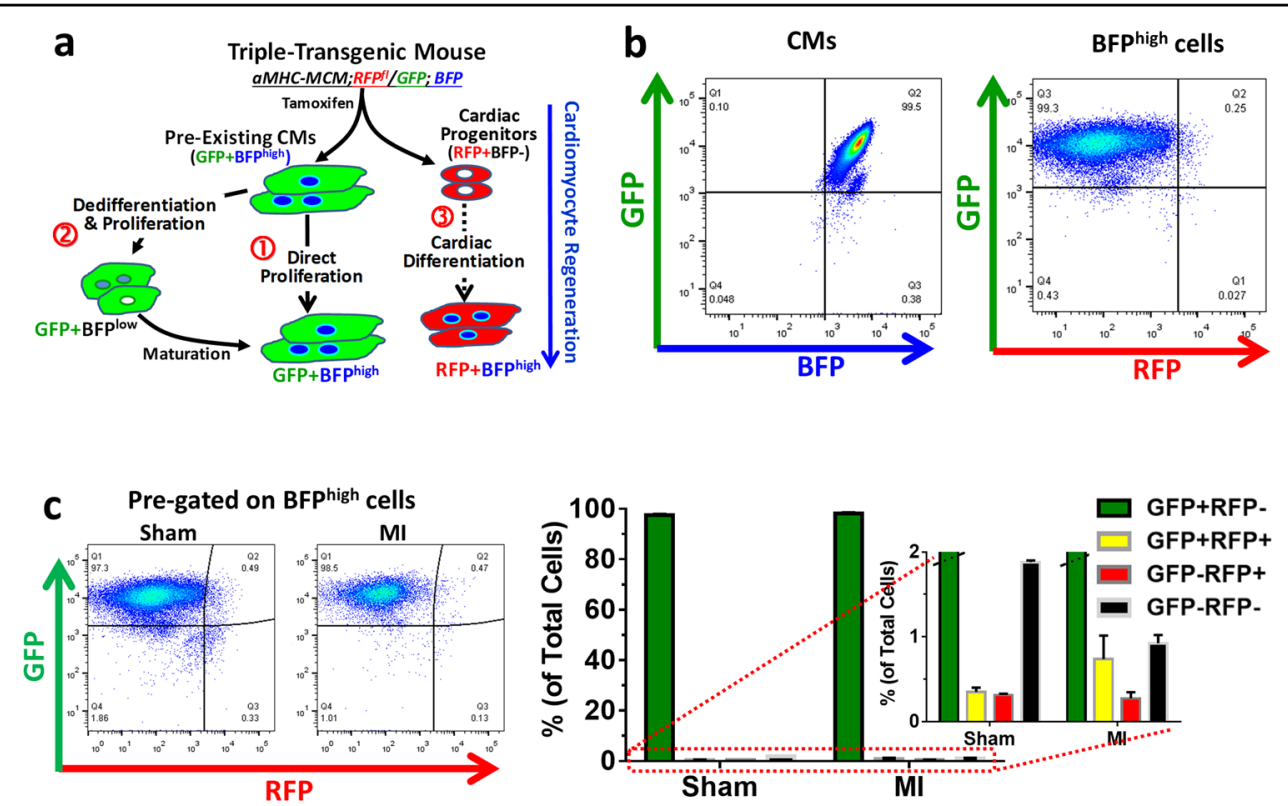

Fig. 3 Adult cardiomyocyte renewal in post-infarct tri-transgenic mouse hearts. a Potential cellular mechanisms underlying endogenous CM renewal in the triple-transgenic mouse model. b Flow cytometry dot plot showing specific expression of both genetic GFP tag and BFP reporter in CM from mice after tamoxifen-induced gene recombination. Nuclei were stained with Draq5. The shown cell dot plot is pre-gated on BFPhigh population. c Characterization of cell populations in tri-transgenic mouse hearts 1.5 weeks after Sham or myocardial infarction (MI). Nuclei were stained with Draq5. No significant changes in BFP myocytes with GFP or RFP reporter. $n=3$ mice (Sham or MI)

areas similar to that in sham-operated hearts (Fig. 5e). These results suggest that $\mathrm{CM}$ dedifferentiation and cell cycle activity might be regulated locally in response to post-infarct remodeling of the myocardium ${ }^{35-37}$.

To assess overall CM dedifferentiation and cell cycle activities in the whole heart, we examined individual heart cells from tri-transgenic mice using single-cell ImageStream analysis (Fig. 6a). There was a significant increase (10.8-fold) of BrdU ${ }^{+}$GFP myocytes in post-MI hearts compared to sham hearts (Fig. 6b). The $\mathrm{GFP}^{+} \mathrm{BFP}^{\text {low }}$ ACM population had a significantly higher $\mathrm{BrdU}^{+}$ incorporation rate compared to $\mathrm{GFP}^{+} \mathrm{BFP}^{\text {high }} \mathrm{ACMs}$, indicating a higher rate of cycling in dedifferentiated $\left(\mathrm{GFP}^{+} \mathrm{BFP}^{\text {low }}\right)$ ACMs (Fig. 6c). To determine if this cycling progressed to cytokinesis we examined the expression of Anillin (Anln) and found that there were more $\mathrm{Anln}^{+}$cells among the $\mathrm{BFP}^{\text {low }}$ GFP ACMs compared to that in BFP high GFP ACM population (Fig. 6d). Cytoskeletal structure in cycling CM appeared to be less organized consistent with a dedifferentiated cell state (Supplementary Fig. S12). ACMs cycling estimated by Ki67 expression was 4.6-fold higher in post-infarct hearts compared to sham hearts; and there was a $\sim 2$-fold increase of $\mathrm{Ki}^{+} 7^{+}$cells in $\mathrm{BFP}^{\text {low }} \mathrm{CMs}$ compared to BFP $^{\text {high }} \mathrm{CMs}(0.63 \%$ versus $0.34 \%$ ) (Fig. 6e). In contrast to $\mathrm{Ki}^{-} \mathrm{CMs}$ in normal hearts being mainly binucleated, cycling $\left(\mathrm{Ki} 67^{+}\right) \mathrm{CMs}$ from post-MI hearts were predominately mononucleated (Supplementary Fig. S13).
These data suggest that dedifferentiated (BFP ${ }^{\text {low }}$ ) ACMs are more actively cycling and may be more likely to divide.

\section{Transcriptomic analysis of in vivo cardiomyocyte dedifferentiation}

To dissect transcriptomic reprogramming of dedifferentiated myocytes in post-infarct hearts, we performed massive parallel single-nucleus RNA-sequencing (snRNAseq) using a modified 10x Genomics protocol allowing a targeted analysis of the highly heterogeneous cell populations in the heart. After filtering and data normalization, we obtained high-quality single-nucleus datasets for 22,992 nuclei from post-MI hearts, and 8550 nuclei for control myocardium. Unsupervised graph-based clustering with smart local moving (SLM) algorithm revealed 15 clusters of cells in the control (Fig. 7a), including common cardiac populations: CMs expressing structural genes (Actn2, Myl2, Tnnt2, Tpm1) and ion channel genes (Scn5a, Kcnj3, and Kcnd2); cardiac fibroblasts expressing Col3a1 and Ddr2; endothelial cells expressing Pecam1 (Cd31) and Tie1; smooth muscle cells expressing Mylk, Pde8b, and Rerg; and a small fraction of macrophages (Fig. 7a; Supplementary Fig. S14a, and Supplementary Table S2). In post-infarct hearts, the myocyte nuclei population was reduced, and inflammatory cells such as macrophages (CD45, Ccr5) and B/T cells (CD74, Fcgr2b, Themis) increased (Fig. 7a, b; and Supplementary Table S3 and S4). $B F P$ and GFP transcripts were enriched in the putative 

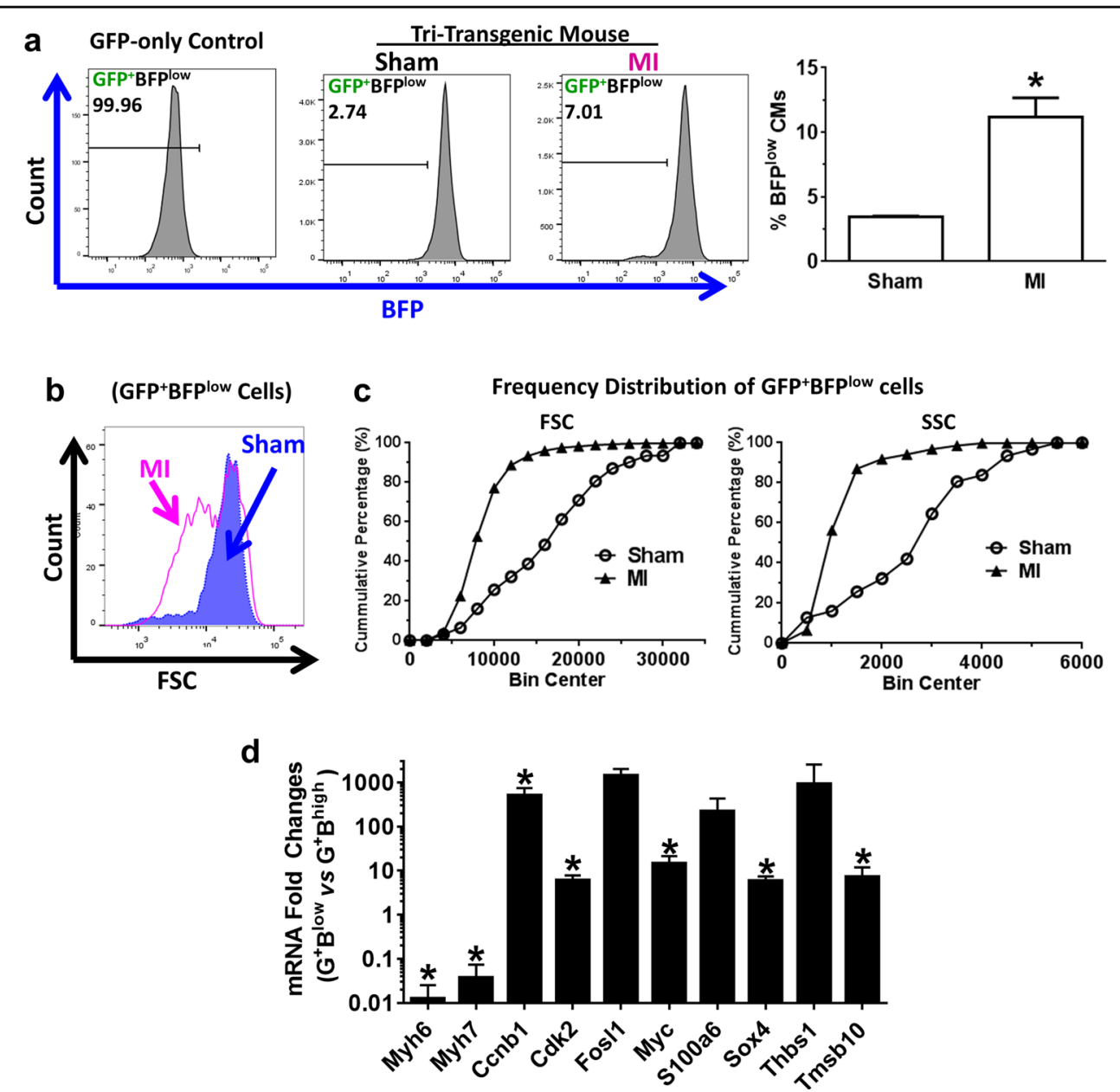

Fig. 4 Increased dedifferentiation GFP myocytes in post-infarct tri-transgenic mouse hearts. a Left panels: flow cytometry histogram. GFP-only control heart cells were used to set the gate. Bar chart: Percentage of BFPlow cells among GFP ${ }^{+}$myocytes in ventricles of 1.5-week post-MI or Sham

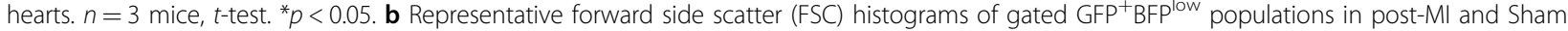
hearts. c Frequency distribution of FSC and side scatter (SSC) indices of GFP ${ }^{+}$BFPlow cells from post-Ml or Sham hearts $(n=3$ mice). One-way ANOVA test: $p<0.0001$ for both FSC and SSC indices, comparing MI to Sham. $\mathbf{d}$ RT-qPCR analysis of gene expression in GFP+ $\mathrm{BFP}^{-}\left(\mathrm{G}^{+} \mathrm{B}^{\text {low }}\right)$ myocytes compared to $\mathrm{GFP}^{+} \mathrm{BFP}^{+}\left(\mathrm{G}^{+} \mathrm{B}^{\text {high }}\right)$ myocytes isolated from post-infarct tri-transgenic mouse hearts. ${ }^{*} p<0.05, \mathrm{G}^{+} \mathrm{B}^{\text {low }} \mathrm{vs}$. $\mathrm{G}^{+} \mathrm{B}^{\text {high }}(n=3$ mice)

CM clusters in both control and post-infarct hearts. The $B F P^{-} / B F P^{+}$ratio of $\mathrm{CM}$ populations $\left(G F P^{+}\right)$increased from $0.93(444 / 475)$ in the control to $9.3(1939 / 209)$ in post-MI hearts (Fig. 7c, and Supplementary Fig. S14b) similar to findings from the flow cytometry analysis (Fig. 4a). While Mki67 was barely detectable in ACM nuclei from the control, there was a significant increase of nuclei expressing $M k i 67$ in the $G F P^{+} B F P^{-}$population in postMI hearts (Fig. 7d, and Supplementary Fig. S15).

In the $\mathrm{GFP}^{+} \mathrm{ACM}$ population, there were 2595 and 1499 DEGs between $B F P^{-}$and $B F P^{+}$nuclei in control and post-infarct hearts, respectively (Supplementary Fig. S16). Compared to $B F P^{+}$nuclei, $B F P^{-}$expressed significantly lower levels of cardiac genes (e.g. Actn2, Cacna1c, Kcnj3, Myh6, Scn5a, Tnnt2), with increases in genes associated with dedifferentiation (Runx1 and Dab2; Fig. 8a) ${ }^{27,31}$. The genes upregulated during $A C M$ in vitro dedifferentiation (Fig. 4d) were also increased in $B F P^{-}$ACM nuclei compared to $B F P^{+}$ones, for example: S100a6 (5.37-fold; $p=$ 0.000355), Tmsb10 (3.81-fold; $p=0.000786)$, Thbs1 (3.45fold, $p=0.0594)$. $B F P^{-}$CM nuclei also expressed higher levels of active cell cycle genes, such as $C c n d 3, C d k 14$ (Fig. 8a). KEGG pathway enrichment analysis on DEGs in $B F P^{-}$(versus $B F P^{+}$) ACMs from post-infarct hearts revealed a number of affected pathways with reduced gene expression, such as those in pathways for cardiac muscle contractile function (Fig. 8b), hypertrophic remodeling, and calcium, adrenergic, and cAMP signaling pathways, and cardiac rhythm (Supplementary Fig. S17a-e; Supplementary Table S5). The expression of genes involved in cardiac metabolic pathways (e.g. pyruvate, TCA cycles) were also reduced in $B F P^{-}$ACMs. However, genes for 


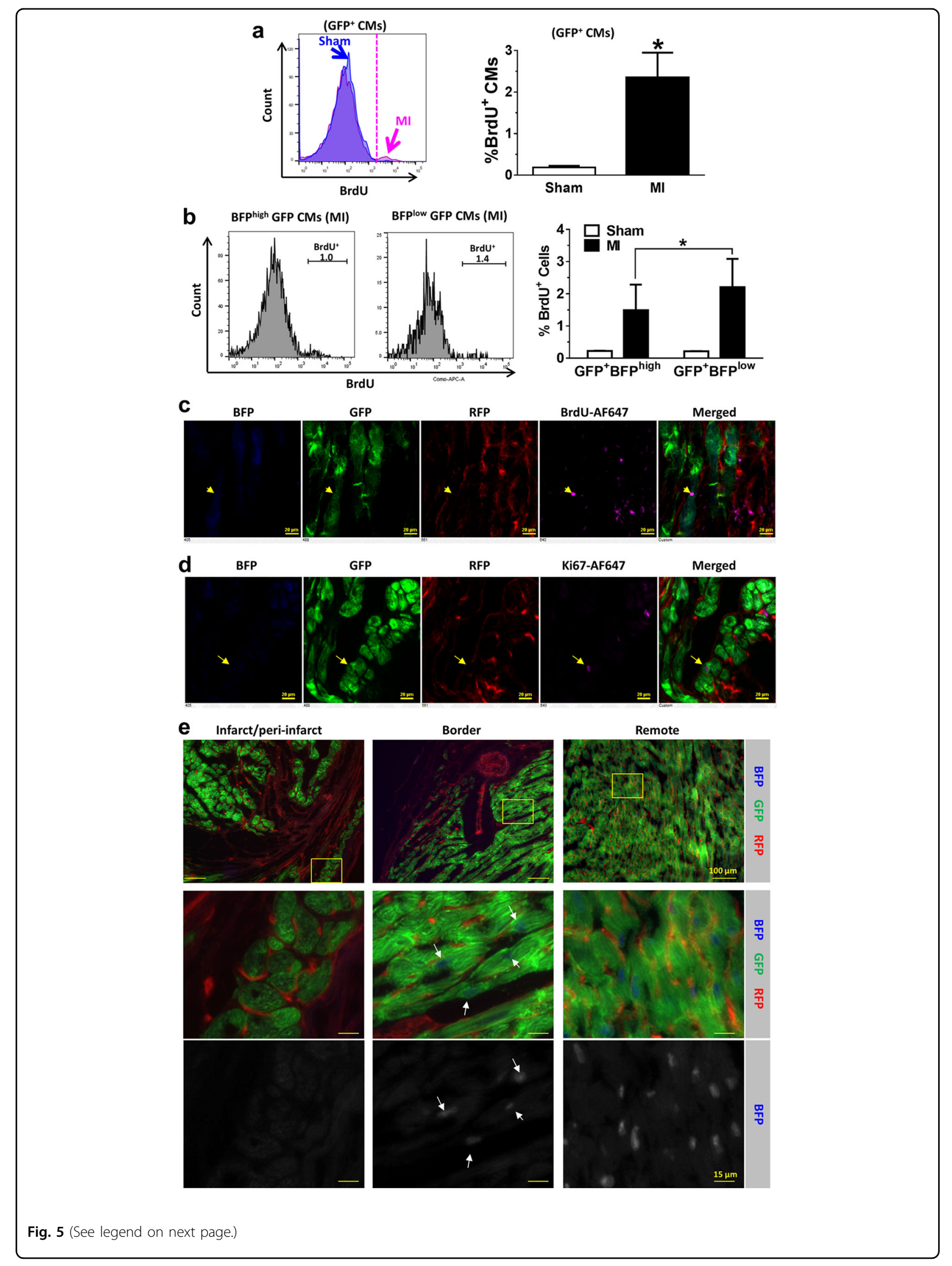


(see figure on previous page)

Fig. 5 Increased cell cycling in dedifferentiated $\mathrm{GFP}^{+}$cardiomyocytes in post-infarct hearts. a and $\mathbf{b}$ Percentages of BrdU $\mathrm{CMs}^{+}$in $(\mathbf{a}) \mathrm{mature}$ myocytes (GFP+BFPhigh) or (b) dedifferentiated myocytes without BFP expression (GFP+BFPlow). ${ }^{*} p<0.05 . n=3$ mice (Sham or MI). $\mathbf{c}$, d BrdU incorporation (c) and Ki67 expression (d) in post-infarct ventricles. Arrows denote the BrdU or Ki67 positive GFP ${ }^{+}$CM. Scale bar $=20 \mu \mathrm{m}$. e Changes in BFP signal in infarct/peri-infarct area, infarct border zone, and remote regions. Boxed regions are shown in middle and lower panels. White arrows show weaker BFP signal in GFP+ cardiomyocytes in the infarct border zone. Scale bar $=100 \mu \mathrm{m}$ for the first row, and $15 \mu \mathrm{m}$ for other rows

several extracellular signaling pathways such as focal adhesion assembly, extracellular matrix receptor interaction, Rap1/Integrin, and survival/proliferation-related PI3K-Akt pathways were up-regulated (Supplementary Fig. 17f-i; Supplementary Table S5). These DEGs together converged on cell survival, dedifferentiation, and proliferation pathways ${ }^{29,30}$. Therefore, the snRNA-seq results indicate that $B F P^{-} \mathrm{ACM}$ populations were molecularly dedifferentiated compared to the $B F P^{+}$ counterparts.

As cycling and proliferative CMs were preferentially found in $\mathrm{BFP}^{-}$population (Figs. 5 and 6), we further examined transcriptomic profiles of $M k i 67^{+}$ACM nuclei in our snRNA-seq datasets. Among the $1939 \mathrm{GFP}^{+} B F P^{-}$ CM nuclei from post-infarct hearts, there were 165 (8.5\%) Mki67-expressing nuclei, which had 681 DEGs when compared to $\mathrm{Ki}^{-}$ACM nuclei (Supplementary Fig. S18). As expected, cell cycle genes such as Anln and the less-studied Knl1 and Kif families were significantly upregulated in $M K i 67^{+}$nuclei of dedifferentiated $\left(B F P^{-}\right)$ CMs (Fig. 8c). Pathway enrichment and gene set enrichment analyses revealed that most of the enriched pathways and processes were related to cell cycle activities, particularly DNA replication, mitosis, cytokinesis, and cell division (Fig. 8d; Supplementary Tables S6 and S7). Furthermore, expression of dedifferentiation genes such as S100a6 and Tmsb10 was higher in $B F P^{-}$(compared to $B F P^{+}$) ACM nuclei, which were also significantly increased in cycling, $M k i 67^{+} \mathrm{ACM}$ nuclei as compared to the Mki67 ones (1.47 and 1.46 fold, respectively; FDR $p$ $<0.05)$. Upstream transcription regulator analysis for the DEGs indicates that S100a6 and Foxm1 are regulators of the transcriptional reprogramming in dedifferentiated and cycling ACMs (Supplementary Fig. S19).

\section{Discussion}

The reported discrepancies in new myocyte formation in injured hearts likely stems from multiple factors. First, the minuscule rate of new $\mathrm{CM}$ formation makes it challenging to measure accurately, and a small variance in quantification can result in markedly different results. Our data also confirmed that endogenous ACM cycling and renewal (e.g. $\mathrm{Ki}_{67}^{+}, \mathrm{Anln}^{+}$, or $\mathrm{BrdU}^{+}$) is at a low rate. Second, significantly higher cycling rates of non-myocytes in the adult heart can lead to inaccurately assigning events to ACM, particularly when not using a genetic tracking model or using one with the incomplete labeling of preexisting CMs. For instance, while MIMS is arguably the most accurate methodology currently available, it relies on the extrapolation of cell identity information derived from adjacent tissue sections ${ }^{8}$. An additional caveat is that conventional analyses dealing with limited numbers of cells may not have the power to reveal the very small number of scant events, i.e. proliferation of pre-existing myocytes or differentiation from putative CPCs (if any). Previous studies using direct genetic labeling of putative rare CPCs, has failed to demonstrate a significant contribution to ACM formation but have been criticized as potential being too narrow in scope, missing a significant contribution of non-myocyte to CM formation ${ }^{21-24}$. In this study, we employed high-throughput and readily implementable technologies, namely flow cytometry and ImageStream, to analyze total cells or total nuclei prepared from the whole ventricle. Conventional flow cytometry is widely available in modern biomedical institutes, enabling rapid data acquisition and analysis, although careful optimization is required when dealing with highly heterogeneous cells such as those in the heart. With spectrum imaging-based flow cytometry, ImageStream, we can capture the image of cell/nucleus, and ensure the rigorous identification of true positives. Instead of focusing on specific areas such as infarct or border zone that can potentially overestimate the response to cardiac injury, we used a non-biased total ventricular cell approach to assess the overall CM cycling activity in mice. Our results using these new transgenic models are the first to provide real-time visualization of CM dedifferentiation and cell cycling. Our data suggest that increased endogenous $\mathrm{CM}$ renewal in post-infarct hearts arise from the dedifferentiation and proliferation of pre-existing CMs and not by cardiac differentiation of putative adult CPCs.

The Cre/LoxP system allows for genetic labeling of cardiac and non-myocyte lineages by the use of conditional gene recombination in a temporal and cell typespecific manner. Many groups, including our own, previously utilized bi-transgenic $\alpha M H C-M C M ; Z / E G$ mouse model to track the fate of CMs that switch on GFP expression after tamoxifen treatment ${ }^{8,28-30}$. However, this transgenic model had an incomplete GFP labeling rate $(\sim 78 \%)$ in pre-existing CMs. Incomplete genetic labeling of cell population could lead to the assignment of events 

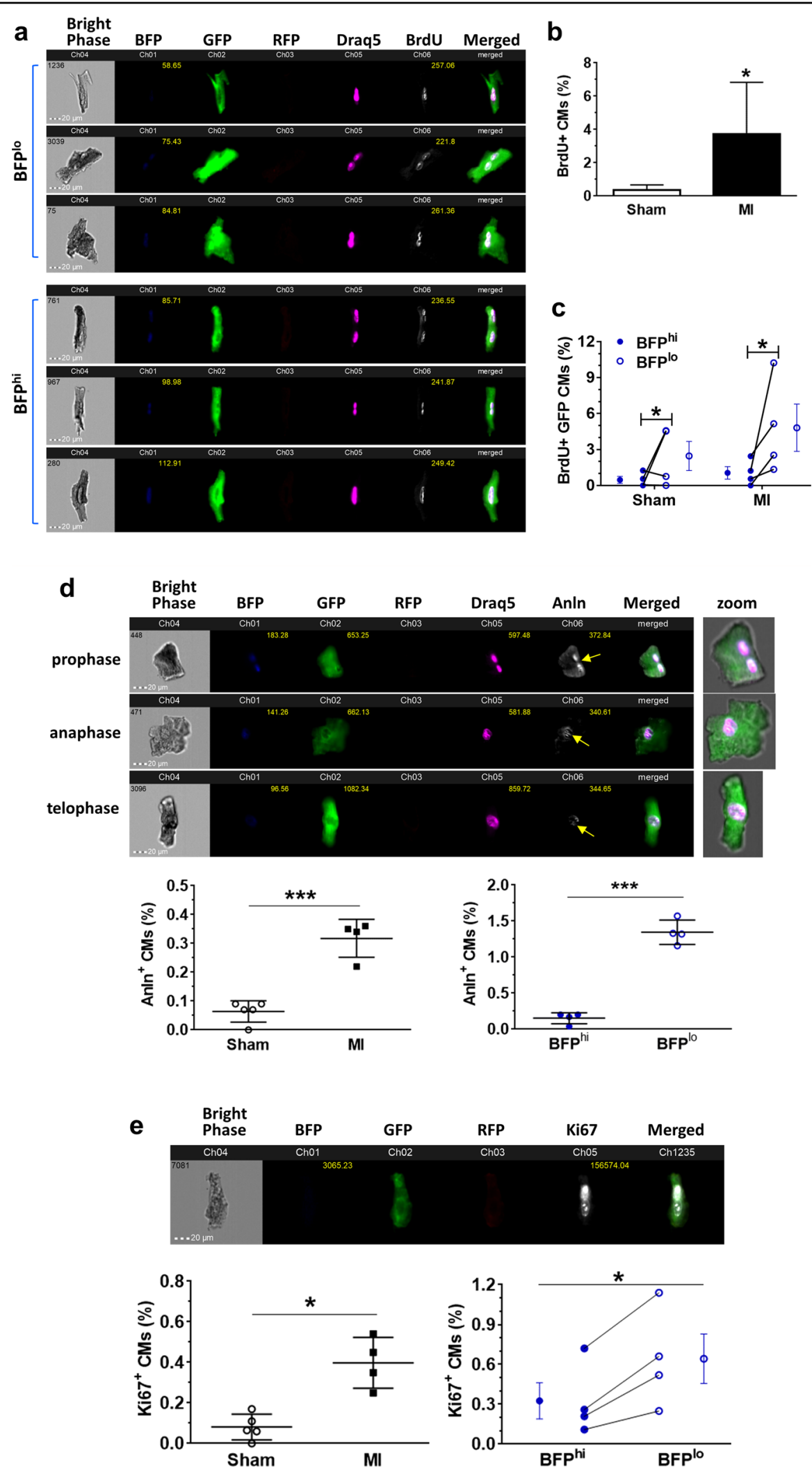

Fig. 6 (See legend on next page.) 
(see figure on previous page)

Fig. 6 Dedifferentiated cardiomyocytes (GFP ${ }^{+}$BFP $^{\text {low }}$ ) demonstrated higher rates of cell cycling. a ImageStream analysis of ventricular cells from tri-transgenic mice. Images demonstrate $\mathrm{GFP}^{+} \mathrm{BrdU}^{+}$myocytes in post-infarct hearts, with BFP and BrdU signals indicated by median pixel intensity. Nuclear BFP signals were classified as no or low (BFPlow) and high (BFPhigh) (see "Materials and methods" for details). b BrdU ${ }^{+}$GFP CM in post-infarct (MI) or sham hearts. ${ }^{*} p<0.05$ by $t$-test. $\mathbf{c}$ BrdU ${ }^{+}$GFP CM in a population with high or low BFP signal (BFPhigh and BFPlow, respectively). $p>$ 0.05 by two-way ANOVA test. ${ }^{*} p<0.05$ by post-hoc Bonferroni test. Connected line represents each mouse. $\mathbf{d}$ ImageStream analysis of anillin (Anln) ${ }^{+}$ CM. Nuclei (stained by Draq5) and AnIn (revealed by APC-Cy7-conjugated antibody) are shown in Ch05 and Ch06, respectively. Yellow arrows denote the mid-body plane. Lower panels show the percentage of Anln+ GFP CM in post-Ml or sham hearts (left), and in BFPhigh or BFPlow GFP CM subpopulations from post-infarct hearts (right). ${ }^{*} p<0.001$ by $t$-test. e ImageStream analysis of Ki67 ${ }^{+} \mathrm{CM}$. Lower panels show the percentage of Ki67 ${ }^{+}$ GFP cardiomyocytes in post-infarct or sham hearts (left), and in the BFP high or BFPlow GFP CM subpopulations from post-infarct hearts (right). ${ }^{*} p<0.05$ by $t$-test. Paired post-infarct hearts identified by connected lines

to other cell types; such errors will affect the accuracy of associated analyses. For a more precise and accurate analysis of cellular activities that occur at such low rates, we developed a more efficient model for CM genetic cell fate mapping. Our data in bi-transgenic $\alpha M H C-M C M$; $\mathrm{RFP}^{\mathrm{fl}} / \mathrm{GFP}$ mice demonstrated highly efficient Cre/LoxP gene recombination that led to the switching of RFP expression to GFP expression in $~ 99 \%$ pre-existing ACMs when animals were fed with tamoxifen chow, a 27\% increase from the previous model. Furthermore, GFP labeling of small, presumably non-myocyte cells in the heart was minimal $(<0.03 \%)$ and showed no significant difference between sham and post-MI hearts (data not shown), confirming the tight control of gene recombination. Thus, we believe the results from this transgenic model more accurately reflect the cell sources of new CM formation in response to pathophysiological stresses such as MI.

We took advantage of the bright BFP variant ${ }^{38}$, and engineered a transgenic mouse with a BFP reporter specific to $\mathrm{CM}$ nuclei. In contrast to the previous $\alpha \mathrm{MHC}$ nLAC model expressing $\beta$-galactosidase specifically in $\mathrm{CM}$ nuclei that requires X-gal staining for visualization ${ }^{39}$, the BFP reporter in our model can be detected with or without immunostaining. This design allows greater flexibility when used in conjunction with other reporter models to detect CM events using standard highthroughput assays such as flow cytometry or high content imaging analysis ${ }^{40,41}$. As cell cycle progression does not affect the H2B-GFP reporter and the $\mathrm{H} 2 \mathrm{~B} /$ reporter fusion protein exchanges at a fast rate ${ }^{42,43}$, we postulated that a reduction in BFP signal reflects lower $\alpha \mathrm{MHC}$ (Myh6) promoter activity in dedifferentiated CMs (Fig. $4 d)^{29}$. Combining both mouse models into a multireporter tri-transgenic line allowed us for the first time to characterize cycling and molecular changes associated with dedifferentiation in vivo (Fig. 3a). The results from both bi-transgenic and tri-transgenic model suggest that pre-existing $\mathrm{CM}$ are the predominate resource of $\mathrm{CM}$ renewal, and are consistent with reports from genetic cell fate tracking of $\mathrm{CPCs}^{21}$. More importantly, we found that $\mathrm{GFP}^{+} \mathrm{BFP}^{\text {low }}$ cells morphologically and molecularly recapitulated the phenotype seen in dedifferentiated CMs. While future studies will focus on the mechanisms to control dedifferentiation, it is interesting to note that many genes up-regulated in dedifferentiated $\mathrm{CMs}$ are transcription factors, for example, Fosl1, Myc, Sox4, and Tmsb10. The Sox family of transcription factors may play a role in the dedifferentiation of multiple cell types and contribute to the reacquisition of primitive cell phenotypes and an enhanced cell cycle activity ${ }^{30,44}$. Therefore, we believe that dedifferentiation (similar to differentiation) and cell cycle activity is a molecularly regulated process in CMs.

Taking the advantages of single-cell transcriptomic analysis, we performed massive parallel cardiac snRNAsequencing analysis that circumvented the issues associated with the significant heterogeneity of adult heart cells and the challenges in sorting a limited amount of dedifferentiated and cycling ACMs. We were able to identify specific cell populations in the heart using distinct transcriptomic clusters, transgenic reporters for ACM lineage and dedifferentiation, as well as cell cycle markers. This data also reveals typical pathological responses in post-infarct myocardium, including activation of fibrotic and inflammatory remodeling that was reflected by a panel of enriched signaling pathways and gene sets. The results demonstrated that the dedifferentiation and cell cycle progression of pre-existing CMs was augmented in post-infarct hearts, consistent with flow cytometry and ImageStream analyses. In the $\mathrm{BFP}^{-}$myocyte population there was expected down-regulations of genes controlling of ACM phenotype and function; but we also discovered a number of new signaling networks that may be potentially specific to ACM dedifferentiation and cell cycle reactivation. For example, the activation of Focal adhesion, Integrin/ECM receptor interaction, Rap1 signaling, and actin cytoskeleton regulation, was seen in $B F P^{-}$ACMs (Supplementary Fig. S17, and Supplementary Tables S5, S6). These pathways play important roles in the dedifferentiation and proliferation of chondrocytes ${ }^{45}$, and the migration and proliferation of vascular smooth muscle cells $^{46,47}$. We also discovered that a subset of up-regulated genes in the $B F P^{-}$and $M K i 67^{+}$ACMs, such as Knl1, 
a
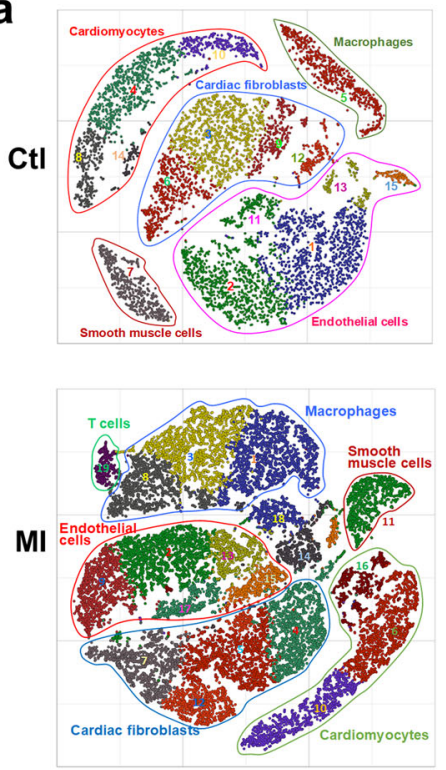

1: Endo (Nav3, Zfip366) - 2: Endo (Flt1, Cyyr1) 4. CM (Konj3. Mhrt) - 5 : Mac (Adgre1, F13a1) - 6. CF (Pcdh9, Bicc1) 7. SM (Pde8b, Rerg) 8: CM (Fst/4, itgb6) - 9: CF (Pi16, Mfap5) - 10: CM (Rbm20, Tnnt2) - 11: Endo (Nrg1, vWF) - 12: CF (Kont2, Podh9) - 14. Cach1, Pecam 1) - 14: CM (Mhrt, Kong2) 3: Mac (Cd86, Fcgr2b) 5. CF (Kif26b, Postn) - 6. CM (Grm1 Mypn)) 6. CM (Grm1. Mypn)) 8: Mac (Fyb, Arhgap15) 9: Endo (Flt1 Ablim3) 10: CM (Mhrt, Kond 3 ) 11: SM (Lin7a, Rerg) - 13: Endo (Ccser1, Col18a1) 15: Endo (Cyyr1, Ldb2) 16: CM (Kond2, Kcnj3) 17: Endo (Npr3, Tmem10) 19: T cells (Skap1, Themis)
- 1: $\operatorname{Mac}(C d 84, C d 300 / f)$ 2. Endo (Rgcc, Adgrl4) 12: CF (Hmcn2, Scara5)

b

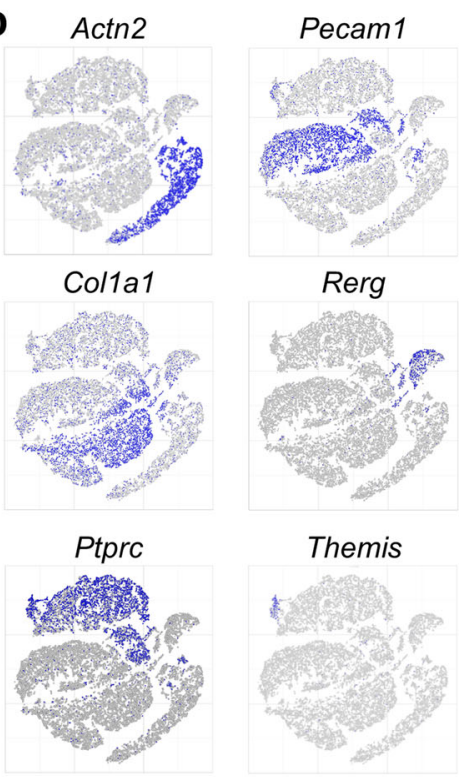

C
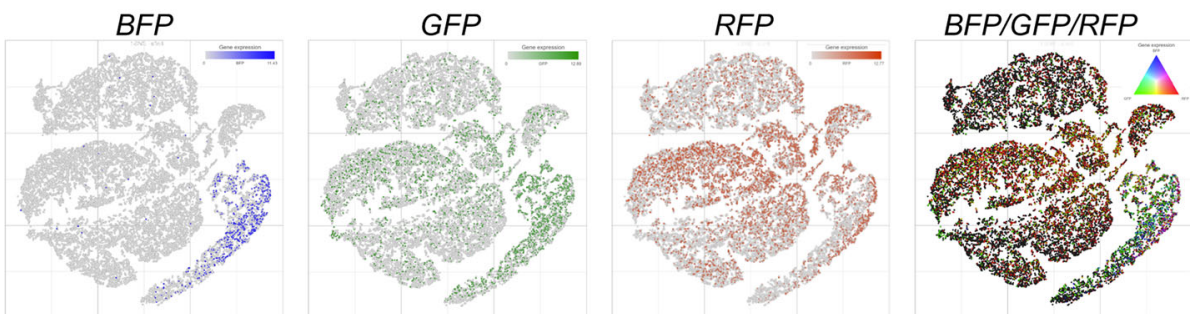

d

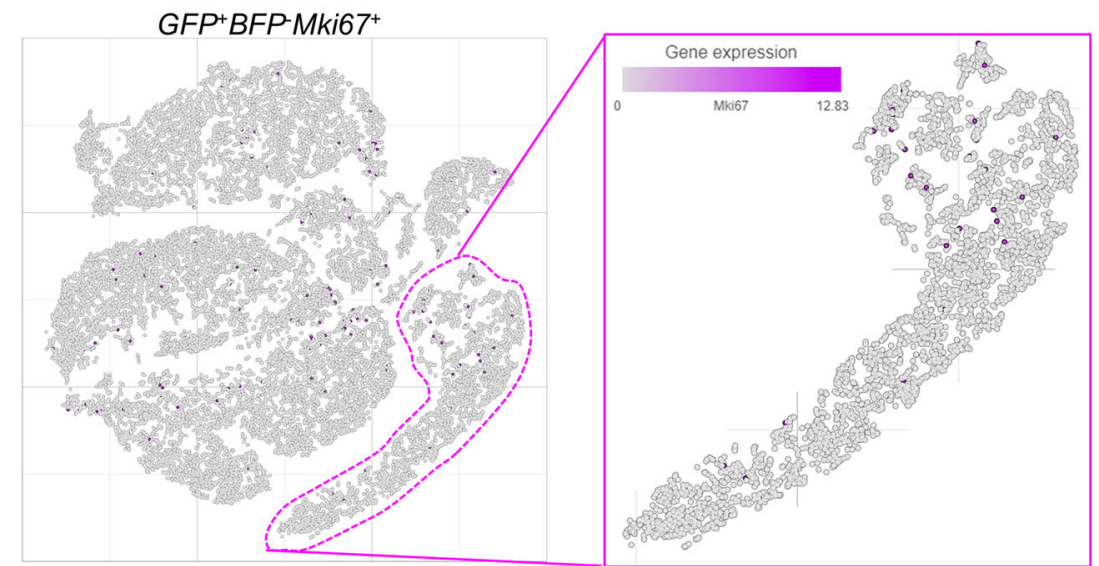

Fig. 7 Single-nucleus RNA-seq reveals specific cell populations in the heart. a t-SNE plots showing graph clustering of heart cells in normal control (Ctl) and post-infarct (MI) hearts. Top-ranked genes differentially expressed in clusters are bracketed. CM cardiomyocytes, CF cardiac fibroblasts, Endo endothelial cells, SM smooth muscle cells, Mac macrophages. b Expression levels of positive expression level (low-gray, high-blue) of markers in myocytes (Actn2), endothelial cells (Pecam1; Cd31), cardiac fibroblasts (Col1a1), smooth muscle cells (Rerg), and macrophages (Ptprc; Cd45), and T cells (Themis) in post-MI hearts. $\mathbf{c}$ Expression of BFP, GFP, and RFP reporter genes in nuclei of post-infarct hearts. $\mathbf{d}$ The t-SNE plot for nuclei from post-infarct hearts showing dedifferentiated cycling CM expressing GFP+ $B F P^{-} M k i 67^{+}$(circled nuclei; Mki67 expression level: low-gray, high-blue). There were $165 \mathrm{Ki}^{6} 7^{+}$nuclei among $1939 \mathrm{BFP}^{-}$ACM nuclei

Kif11, and Cdk14, are known to promote cell cycle and proliferation in other cells. While single-cell (or nucleus) RNA-seq analysis can be limited by the sequencing depth for each cell (nucleus), we found that at least some of the DEGs identified with in vivo single-nucleus RNA-seq analyses overlap with those identified from in vitro $\mathrm{CM}$ 


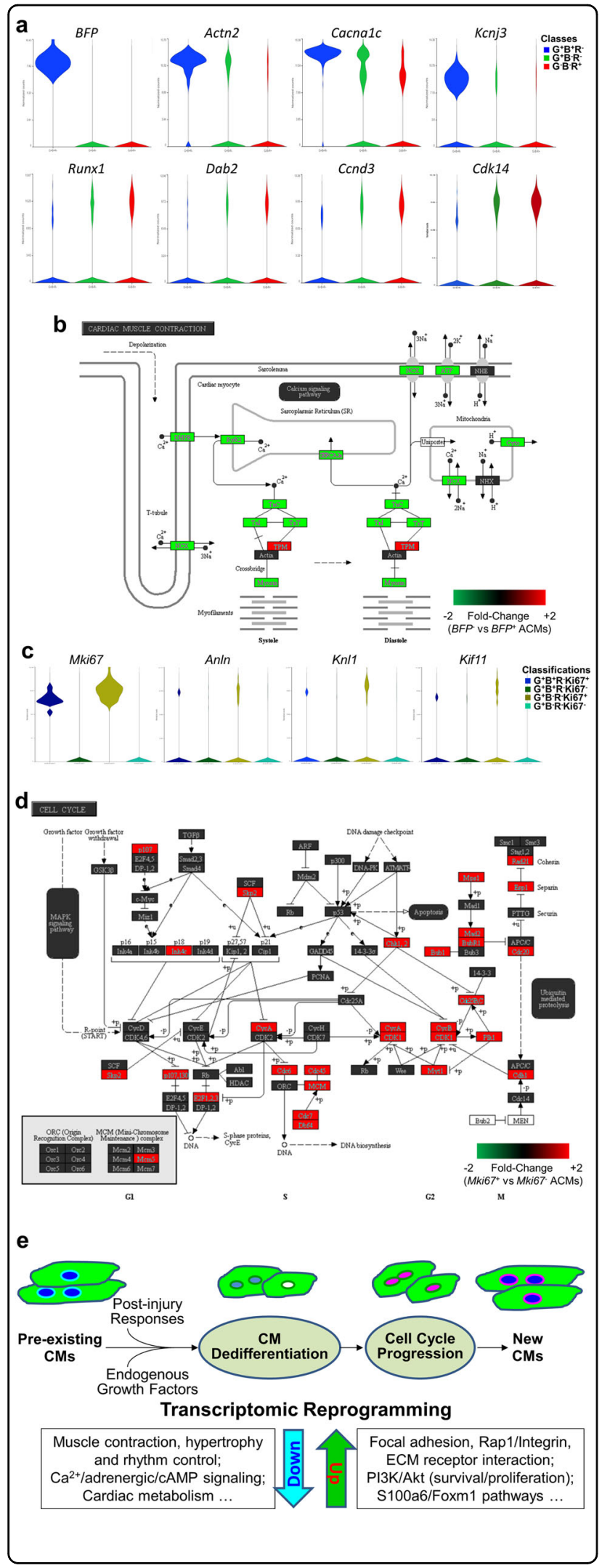

Fig. 8 Cardiomyocyte dedifferentiation and cell cycling revealed by single-nucleus transcriptomes. a Violin plots showing the expression of genes in $G F P^{+} B F P^{+} R F P^{-}\left(\mathrm{G}^{+} \mathrm{B}^{+} \mathrm{R}^{-}\right.$; blue), GFP+ $B F P^{-} R F P^{-}$ $\left(\mathrm{G}^{+} \mathrm{B}^{-} \mathrm{R}^{-}\right.$; green), and $G F P^{-} B F P^{-} R F P^{+}\left(\mathrm{G}^{-} \mathrm{B}^{-} \mathrm{R}^{+}\right.$; red) nuclei from postinfarct hearts. Normalized expression values for these genes had a | Fold-change| $>2$ with a FDR, $p \leq 0.01$. $\mathbf{b}$ Enrichment of Cardiac muscle contraction pathway (enrichment score 10.64, p 2.39E-05) by the DEGs (|Fold-change| $>2$; FDR, $p \leq 0.01$ ) between $B F P^{-}$and $B F P^{+} C M$ nuclei $\left(G F P^{+} R F P^{-}\right)$from post-infarct hearts. c Expression of cell cycle genes in normal or dedifferentiated cardiomyocytes nuclei from postinfarct hearts. $\mathbf{d}$ Enrichment of cell cycle pathway (enrichment score 36.974; $\mathrm{p} 8.76 \mathrm{E}-17$ ) by the DEGs (|fold-change| $>2$; FDR $p \leq 0.01$ ) between $M M^{2} i 67^{+}$and $M k i 67^{-}$dedifferentiated cardiomyocyte nuclei $\left(G F P^{+} B F P^{-} R F P^{-}\right)$from post-infarct hearts. e Working model of CM regeneration in vivo

dedifferentiation including S100a6 and Tmsb10. Hence, targeting the related S100a6/Foxm1 signaling pathways may promote ACM dedifferentiation and cell proliferation. One caveat with cardiac single-nucleus analysis is the assumption that the nuclei within bi-nucleated or trinucleated myocytes are highly similar in their gene expression. The tight clustering of myocyte nuclear populations and their distinction from non-CM populations, suggests this is a reasonable assumption.

In summary, we believe that transcriptomic reprogramming, including the inactivation of gene networks governing ACM phenotype and function, together with the activation of de novo pathways and transcription factors, ultimately lead to the dedifferentiation and cell cycle progression in pre-existing CMs, giving rise to new $\mathrm{CM}$ formation (Fig. 8e). Given that prolonged cardiomyocyte dedifferentiation can adversely affect cardiac function ${ }^{27}$, knowledge of the specific regulators of both dedifferentiation and cell cycle reactivation will be required if this process is to be exploited therapeutically to promote endogenous $\mathrm{CM}$ proliferation without diminishing heart function.

\section{Materials and methods}

All animals were maintained and experiments were performed in accordance with the guidelines outlined in the Public Health Service Policy on the Humane Care and Use of Laboratory Animals. Animal studies were performed under the protocol approved by the Institutional Animal Care and Use Committee at the University of Washington.

All transcriptomic data have been made publicly available at Gene Expression Omnibus under SuperSeries GSE129175, and at the ArrayExpress portal with accession number E-MTAB-3981, or from the corresponding author upon request.

The detailed "Materials and methods" section is available in the online Supplementary Information. 


\section{Acknowledgements}

This work was supported in part by grants from the American Heart and Stroke Foundation (15BGIA22410026 and 18IPA34110210 to Y.Z.), the US National. Institute of Health (NIH) (R56HL131898 to Y.Z., and R01HL070748 to W.R.M.), and the research awards from John Locke Trust Charitable Foundation (to Y.Z. and W.R.M.). The authors thank Yi Lin, Jingyi Morgan Wong, Sahir Jabbouri, and Yasi Zhong for technical assistance in animal experiments, microscopy imaging, and data analysis. We acknowledge the support of the Cell Analysis Facility Flow and Imaging Cytometry Core Lab and the Garvey Imaging Core, and the Fred Hutch Cancer Center Genomics Core. The authors are also thankful for having the comments and input from Drs. April Stempien-Otero, David Dichek, Charles Murry and Michael T. Chin, and the members of MacLellan lab group.

\section{Author details}

'Division of Cardiology, Department of Medicine, University of Washington, Seattle, WA, USA. ${ }^{2}$ Center for Cardiovascular Biology, University of Washington, Seattle, WA, USA. Institute for Stem Cell and Regenerative Medicine, University of Washington, Seattle, WA, USA. ${ }^{4}$ State Key Laboratory for Biology of Plant Diseases and Insect Pests, Institute of Plant Protection, Chinese Academy of Agricultural Sciences, Beijing, China. ${ }^{5}$ Department of Pathology, University of Washington, Seattle, WA, USA. ${ }^{6}$ Department of Bioengineering, University of Washington, Seattle, WA, USA

\section{Author contributions}

Y.Z. conceived the study and performed project planning, experimental design, generation of transgenic models, in vivo heart cell renewal analysis, ACM cell culture and characterizations, flow cytometry and ImageStream analyses, histology, GPCR, transcriptomic analysis for multiple types of CMs, single-nucleus RNA-seq for tri-transgenic mouse hearts, data analysis, and wrote the manuscript. N.G.-L. performed analyses on the bi-transgenic model. Y.L. and A.M.M. performed animal surgeries and pathological analysis. N.G.-L., N.L., Z.Z., N.A., and W.R.M. performed data analysis. N.A. and A.M. performed genotyping of animals and BFP expression in heart tissues. Y.Z. and W.R.M. conceptualized the study, provided the funding support, and wrote the manuscript with contributions by all authors.

\section{Conflict of interest}

The authors declare that they have no conflict of interest.

\section{Publisher's note}

Springer Nature remains neutral with regard to jurisdictional claims in published maps and institutional affiliations.

Supplementary Information accompanies the paper at (https://doi.org/ 10.1038/s41421-019-0095-9).

Received: 31 July 2018 Revised: 1 April 2019 Accepted: 1 April 2019 Published online: 04 June 2019

\section{References}

1. Porrello, E. R. et al. Transient regenerative potential of the neonatal mouse heart. Science 331, 1078-1080 (2011).

2. Aurora, A. B. et al. Macrophages are required for neonatal heart regeneration. J. Clin. Invest. 124, 1382-1392 (2014).

3. Porrello, E. R. et al. miR-15 family regulates postnatal mitotic arrest of cardiomyocytes. Circ. Res. 109, 670-679 (2011).

4. Jesty, S. A. et al. c-kit+ precursors support postinfarction myogenesis in the neonatal, but not adult, heart. Proc. Natl Acad. Sci. USA 109, 13380-13385 (2012).

5. Ali, S. R. et al. Existing cardiomyocytes generate cardiomyocytes at a low rate after birth in mice. Proc. Natl Acad. Sci. USA 111, 8850-8855 (2014).

6. Bergmann, O. et al. Evidence for cardiomyocyte renewal in humans. Science 324, 98-102 (2009).

7. Mollova, M. et al. Cardiomyocyte proliferation contributes to heart growth in young humans. Proc. Natl Acad. Sci. USA 110, 1446-1451 (2013).

8. Senyo, S. E. et al. Mammalian heart renewal by pre-existing cardiomyocytes. Nature 493, 433-436 (2013).
9. Canseco, D. C. et al. Human ventricular unloading induces cardiomyocyte proliferation. J. Am. Coll. Cardiol. 65, 892-900 (2015).

10. Sereti, K. I. et al. Analysis of cardiomyocyte clonal expansion during mouse heart development and injury. Nat. Commun. 9, 754 (2018).

11. Bergmann, O. et al. Dynamics of cell generation and turnover in the human heart. Cell 161, 1566-1575 (2015).

12. Zhang, Y., Mignone, J. \& MacLellan, W. R. Cardiac regeneration and stem cells. Physiol. Rev. 95, 1189-1204 (2015).

13. Eschenhagen, T. et al. Cardiomyocyte regeneration: a consensus statement. Circulation 136, 680-686 (2017).

14. Li, Y. et al. Genetic lineage tracing of nonmyocyte population by dual recombinases. Circulation 138, 793-805 (2018).

15. Smith, R. R. et al. Regenerative potential of cardiosphere-derived cells expanded from percutaneous endomyocardial biopsy specimens. Circulation 115, 896-908 (2007).

16. Fransioli, J. et al. Evolution of the c-kit-positive cell response to pathological challenge in the myocardium. Stem Cells 26, 1315-1324 (2008).

17. Tateishi, K. et al. Clonally amplified cardiac stem cells are regulated by Sca-1 signaling for efficient cardiovascular regeneration. J. Cell Sci. 120, 1791-1800 (2007).

18. Ellison, G. M. et al. Adult c-kit(pos) cardiac stem cells are necessary and sufficient for functional cardiac regeneration and repair. Cell 154, 827-842 (2013).

19. Uchida, S. et al. Sca1-derived cells are a source of myocardial renewal in the murine adult heart. Stem Cell Rep. 1, 397-410 (2013).

20. Henning, R. J. Stem cells for cardiac repair: problems and possibilities. Future Cardiol. 9, 875-884 (2013).

21. van Berlo, J. H. et al. c-kit+ cells minimally contribute cardiomyocytes to the heart. Nature 509, 337-341 (2014).

22. $\mathrm{He}$, $\mathrm{L}$. et al. Enhancing the precision of genetic lineage tracing using dual recombinases. Nat. Med. 23, 1488-1498 (2017).

23. Liu, Q. et al. Genetic lineage tracing identifies in situ Kit-expressing cardiomyocytes. Cell Res. 26, 119-130 (2016).

24. Sultana, N. et al. Resident c-kit(+) cells in the heart are not cardiac stem cells. Nat. Commun. 6, 8701 (2015).

25. Muzumdar, M. D., Tasic, B., Miyamichi, K., Li, L. \& Luo, L. A global doublefluorescent Cre reporter mouse. Genesis 45, 593-605 (2007).

26. Sohal, D. S. et al. Temporally regulated and tissue-specific gene manipulations in the adult and embryonic heart using a tamoxifen-inducible Cre protein. Circ. Res. 89, 20-25 (2001).

27. Kubin, $T$. et al. Oncostatin $M$ is a major mediator of cardiomyocyte dedifferentiation and remodeling. Cell Stem Cell 9, 420-432 (2011).

28. Malliaras, K. et al. Cardiomyocyte proliferation and progenitor cell recruitment underlie therapeutic regeneration after myocardial infarction in the adult mouse heart. EMBO Mol. Med. 5, 191-209 (2013).

29. Zhang, Y. et al. Dedifferentiation and proliferation of mammalian cardiomyocytes. PloS One 5, e12559 (2010).

30. Zhang, Y. et al. Epigenomic reprogramming of adult cardiomyocyte-derived cardiac progenitor cells. Sci. Rep. 5, 17686 (2015).

31. Wang, W. E. et al. Dedifferentiation, proliferation, and redifferentiation of adult mammalian cardiomyocytes after ischemic injury. Circulation 136, 834-848 (2017).

32. D'Uva, G. et al. ERBB2 triggers mammalian heart regeneration by promoting cardiomyocyte dedifferentiation and proliferation. Nat. Cell Biol. 17, 627-638 (2015).

33. Kuhn, B. et al. Periostin induces proliferation of differentiated cardiomyocytes and promotes cardiac repair. Nat. Med. 13, 962-969 (2007).

34. Meselson, M. S. \& Radding, C. M. A general model for genetic recombination. Proc. Natl Acad. Sci. USA 72, 358-361 (1975).

35. French, B. A. \& Kramer, C. M. Mechanisms of post-infarct left ventricular remodeling. Drug Discov. Today Dis. Mech. 4, 185-196 (2007).

36. Nian, M., Lee, P., Khaper, N. \& Liu, P. Inflammatory cytokines and postmyocardial infarction remodeling. Circ. Res. 94, 1543-1553 (2004).

37. Sun, Y. \& Weber, K. T. Infarct scar: a dynamic tissue. Cardiovasc. Res. 46, 250-256 (2000).

38. Mena, M. A., Treynor, T. P., Mayo, S. L. \& Daugherty, P. S. Blue fluorescent proteins with enhanced brightness and photostability from a structurally targeted library. Nat. Biotechnol. 24, 1569-1571 (2006).

39. Soonpaa, M. H., Koh, G. Y., Klug, M. G. \& Field, L. J. Formation of nascent intercalated disks between grafted fetal cardiomyocytes and host myocardium. Science 264, 98-101 (1994). 
40. Hirai, M., Chen, J. \& Evans, S. M. Tissue-specific cell cycle indicator reveals unexpected findings for cardiac myocyte proliferation. Circ. Res. 118, 20-28 (2016).

41. Klochendler, A. et al. A transgenic mouse marking live replicating cells reveals in vivo transcriptional program of proliferation. Dev. Cell 23, 681-690 (2012).

42. Kanda, T., Sullivan, K. F. \& Wahl, G. M. Histone-GFP fusion protein enables sensitive analysis of chromosome dynamics in living mammalian cells. Curr. Biol. 8, 377-385 (1998).

43. Kimura, H. \& Cook, P. R. Kinetics of core histones in living human cells: little exchange of $\mathrm{H} 3$ and $\mathrm{H} 4$ and some rapid exchange of H2B. J. Cell Biol. 153, 1341-1353 (2001)
44. Negi, S. et al. Analysis of beta-cell gene expression reveals inflammatory signaling and evidence of dedifferentiation following human islet isolation and culture. Plos One 7, e30415 (2012).

45. Shin, $H$. et al. Focal adhesion assembly induces phenotypic changes and dedifferentiation in chondrocytes. J. Cell Physiol. 231, 1822-1831 (2016).

46. Tang, D. D. \& Gerlach, B. D. The roles and regulation of the actin cytoskeleton, intermediate filaments and microtubules in smooth muscle cell migration. Respir. Res. 18, 54 (2017).

47. Li, Q. et al. Rap1 promotes proliferation and migration of vascular smooth muscle cell via the ERK pathway. Pathol. Res. Pract. 214, 1045-1050 (2018). 